Pacific Journal of Mathematics

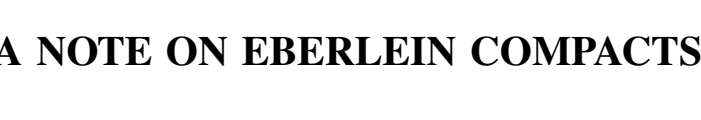




\title{
A NOTE ON EBERLEIN COMPACTS
}

\author{
E. Michael AND M. E. Rudin
}

\begin{abstract}
A new proof is given of the theorem that every continuous image of an Eberlein compact is an Eberlein compact, and a new characterization of Eberlein compacts is obtained.
\end{abstract}

1. Introduction. According to D. Amir and J. Lindenstrauss [1], an Eberlein compact, or EC, is a space ${ }^{1)}$ which is homeomorphic to a weakly compact (i.e., compact in the weak topology) subset of a Banach space. The following theorem, which had been conjectured by Lindestrauss in [6, Problem 5], was proved in [2, Theorem 2.1] by Y. Benyamini, M. E. Rudin and M. Wage.

THEOREM 1.1. [2]. Every continuous image of an EC is again an $E C$.

The proof of Theorem 1.1 in [2] uses a Banach space characterization of $E C$ 's due to Amir and Lindestrauss [1, Theorem 1] (see Theorem 1.4 (b) below), as well as a purely topological characterization-based on the former-which was obtained by H. P. Rosenthal in [9, Theorem 3.1] (see Theorem 1.4 (d)). The main purpose of this note is to give a somewhat more direct proof of Theorem 1.1 which depends only on Rosenthal's characterization, and which yields a new topological characterization of EC's-formally weaker than Rosenthal's-which is given in Theorem 1.4 (e).

Before stating Theorem 1.4, we need two definitions.

Definition 1.2. A collection $\mathscr{U}$ of subsets of $X$ is point-finite if each $x \in X$ is in only finitely many $U \in \mathscr{C}$; it is $\sigma$-point-finite if $\mathscr{U}=\bigcup_{n=1}^{\infty} \mathscr{U}_{n}$ with each $\mathscr{U}_{n}$ point-finite.

DEFINITION 1.3. A collection $\mathscr{U}$ of subsets of $X$ separates (resp. F-separates) $X$ if, whenever $x \neq x^{\prime}$ are in $X$, then there is a $U \in \mathscr{Q}$ such that $x \in U$ and $x^{\prime} \notin U$ (resp. $x \in U$ and $x^{\prime} \notin \bar{U}$ ), or vice versa. ${ }^{2)}$

In the following theorem, $c_{0}(\Gamma)$ denotes the Banach space of real-valued functions vanishing at infinity on the set $\Gamma$, and $\mathscr{T}_{w}$

1 All spaces in this paper are Hausdorff.

2 Many authors use the term " $\mathscr{U}$ separates $X$ " to denote the preceding concept without the phrase "or vice versa." In this paper, a collection $\mathscr{U}$ with this stronger property is called strongly separating (see (1.6)). It should be remarked that, unlike strongly separating collections, separating collections need not be coverings. 
(resp. $\mathscr{T}_{p}$ ) the weak topology (resp. topology of pointwise convergence) on $c_{0}(\Gamma)$.

THEOREM 1.4. The following properties of a compact space $X$ are equivalent.

(a) $X$ is an EC.

(b) $X$ is homeomorphic to a subset of some $\left(c_{0}(\Gamma), \mathscr{T}_{w}\right)$.

(c) $X$ is homeomorphic to a subset of some $\left(c_{0}(\Gamma), \mathscr{T}_{p}\right)$.

(d) $X$ has a $\sigma$-point-finite, separating collection of open $F_{\sigma^{-}}$ subsets.

(e) $X$ has a $\sigma$-point-finite, $F$-separating collection of open subsets.

All of Theorem 1.4, except (d) $\leftrightarrow(\mathrm{e})$, is known. For details see $\S 2$, where we also give a proof of the equivalence of (b) and (d) (via (c)) which is somewhat more direct than Rosenthal's in [9].

The following remarks explain the significance of the requirements in parts (d) and (e) of Theorem 1.4.

(1.5). The $F_{\sigma}$ requirement cannot be omitted from 1.4 (d) (equivalently: " $F$-separating" cannot be weakened to "separating" in $1.4(\mathrm{e}))$. In fact, there exists a compact space with a countable, separating open cover which is not an $E C$ [10, Remark, p. 183] $[11$, p. 20]. By contrast, every compact space with a point-finite, separating collection of open subsets is an EC [10, Proposition 9], and a compact space with a countable, F-separating collection of open subsets must even be metrizable (by 1.6 below).

(1.6). Every compact space with a point-countable, strongly separating (see footnote 2 ) open cover is metrizable [8].

(1.7). It is consistent with $Z F C$ that " $\sigma$-point-finite" cannot be weakened to "point-countable" in $1.4(\mathrm{~d})$ and (e) [2, Example 5.1].

We conclude this introduction with a question. A positive answer would (in view of Theorem 1.4) provide a significant generalization of Theorem 1.1.

Problem 1.8. Suppose $X$ is a normal space which is homeomorphic to a subset of some $\left(c_{0}(\Gamma), \mathscr{T}_{p}\right)$. Must every image of $X$ under a perfect map be homeomorphic to a subset of some $\left(c_{0}(\Gamma), \mathscr{T}_{p}\right)$ ?

The paper is arranged as follows. Section 2 proves parts of Theorem 1.4. Section 3 contains some lemmas which are needed in the proof Theorem 1.1, $\S 4$ proves Theorem 1.1, and $\S 5$ considers 
additional consequences of this proof. Section 6 deals with a modification of $E C$ 's.

2. Proof of Theorem 1.4. As observed in the introduction, the only part of this theorem which is new is the equivalence $(d) \leftrightarrow$ (e). The simple and straightforward proofs for (c) $\rightarrow(b)$ and (c) $\leftrightarrow$ (d) given below are known (although the one for (c) $\leftrightarrow$ (d) does not seem to have appeared in print), and we include them here for the sake of completeness.

(a) $\rightarrow(b)$. This is a deep result of Amir and Lindenstrauss [1, Theorem 1].

(b) $\rightarrow$ (a). Clear.

(b) $\rightarrow$ (c). Clear, since $\mathscr{T}_{w}$ is finer than $\mathscr{T}_{p}$.

(c) $\rightarrow(\mathrm{b})$. Suppose $X \subset\left(c_{0}(\Gamma), \mathscr{T}_{p}\right)$ is compact. Then $X$ is homeomorphic (via the map $f \rightarrow(\arctan ) \circ f)$ to a bounded compact $Y \subset$ $\left(c_{0}(\Gamma), \mathscr{T}_{p}\right)$, and on such a $Y$ the topologies $\mathscr{T}_{p}$ and $\mathscr{T}_{w}$ must agree by a theorem of A. Grothendieck [5, Theorem 5] (applied to the one-point compactification of the discrete space $\Gamma$ ).

$(\mathrm{c}) \rightarrow(\mathrm{d})$. It will suffice to show that $c_{0}(\Gamma)$ has a $\sigma$-point-finite, separating collection $\mathscr{U}$ of open $F_{\sigma}$-subsets. For $r \neq 0$ and $\gamma \in \Gamma$, let $U_{r, r}=\left\{f \in c_{0}(\Gamma): f(\gamma)>r\right\}$ if $r>0$ and let $U_{r, r}=\left\{f \in c_{0}(\Gamma): f(\gamma)<r\right\}$ if $r<0$. Let $\mathscr{C}_{r}=\left\{U_{r, r}: \gamma \in \Gamma\right\}$, and let $\mathscr{U}_{L}=\bigcup\left\{\mathscr{U}_{r}: r \neq 0, r\right.$ rational $\}$. This $\mathscr{U}$ has the required properties.

(d) $\rightarrow$ (c). Let $\mathscr{U}_{6}=\bigcup_{n=1}^{\infty} \mathscr{U}_{n}$ be a separating collection of open $F_{\sigma}$-subsets of $X$, with each $\mathscr{U}_{n}$ point-finite and with $\mathscr{U}_{n} \cap \mathscr{U}_{m}=\varnothing$ if $n \neq m$. For each $U \in \mathscr{C}_{n}$, choose a continuous $f_{U}: X \rightarrow[0,1 / n]$ such that $f^{-1}(0)=X-U$. Let $\Gamma=\mathscr{Q}$. For each $x \in X$, define $\widetilde{x} \in c_{0}(\Gamma)$ by $\widetilde{x}(U)=f_{U}(x)$. Then $x \rightarrow \widetilde{x}$ is a homeomorphism from $X$ into $c_{0}(\Gamma)$.

$(\mathrm{d}) \rightarrow(\mathrm{e})$. Clear.

$(e) \rightarrow(f)$. This will be established by our proof of Theorem 1.1.

\section{Four lemmas.}

Lemma 3.1 (D. Burke [3, Lemma 4.1]). Let $\mathscr{P}$ be a pointfinite collection of subsets of $X$, let $E \subset X$ and let $n \in N$. Then there are at most finitely many minimal covers $\mathscr{F}$ of $E$ such that $\mathscr{F} \subset \mathscr{P}$ and $|\mathscr{F}|=n$.

Proof. (Included for completeness.) Suppose there were an infinite family $\Phi$ of such $\mathscr{F}$. Pick a maximal $\mathscr{R} \subset \mathscr{P}$ such that $\mathscr{R} \subset \mathscr{F}$ for infinitely many $\mathscr{F} \in \Phi$, and let $\Phi^{\prime}=\{\mathscr{F} \in \Phi: \mathscr{F} \supset \mathscr{R}\}$. Clearly $0 \leqq|\mathscr{R}|<n$, so $\mathscr{R}$ does not cover $E$ and hence there is some $y \in E-\bigcup \mathscr{R}$. Since $\mathscr{P}$ is point-finite and $\Phi^{\prime}$ is infinite, there 
is some $P_{0} \in \mathscr{P}$ such that $y \in P_{0}$ and $P_{0} \in \mathscr{F}$ for infinitely many $\mathscr{F} \in \Phi^{\prime}$. Since $y \in P_{0}$, we have $P_{0} \notin \mathscr{R}$. Let $\mathscr{S}=\mathscr{R} \cup\left\{P_{0}\right\}$. Then $\mathscr{R} \neq \mathscr{S}$ and $\mathscr{S} \subset \mathscr{F}$ for infinitely many $\mathscr{F} \in \Phi$, contradicting the maximality of $\mathscr{R}$.

LEMMA 3.2. Let $Y$ be completely regular, let $\lambda=|Y|$, and let $V \subset Y$ be open. Then there is an open cover $\left\{V_{\alpha}: \alpha<\lambda\right\}$ of $V$, and a closed (in $Y$ ) cover $\left\{S_{\alpha, m}: \alpha<\lambda, m \in N\right\}$ of $V$, such that:

(a) $S_{\alpha, m} \subset V_{\alpha} \subset V$ for all $\alpha, m$.

(b) If $\alpha>\beta$, then $S_{\alpha, m} \cap V_{\beta}=\varnothing$ for all $m$.

Proof. Let $\left\{V_{\alpha}: \alpha<\lambda\right\}$ be a cover of $V$ by open $F_{\sigma}$-subsets of $Y$, so $V_{\alpha}=\bigcup_{m=1}^{\infty} T_{\alpha, m}$ with each $T_{\alpha, m}$ closed in $Y$. For each $\alpha, m$, let

$$
S_{\alpha, m}=T_{\alpha, m}-\bigcup_{\beta<\alpha} V_{\beta} \text {. }
$$

It is easy to check that this works.

LEMma 3.3. Let $\mathscr{Q}$ be an $F$-separating open cover of a space $X$, and $A, B$ disjoint compact subsets of $X$. Then there is a finite $\mathscr{E} \subset \mathscr{U}$ which $F$-separates any $x \in A$ from any $x^{\prime} \in B$ (in the obvious sense).

Proof. Since $\mathscr{U}$ is $F$-separating and $A \cap B=\varnothing$,

$$
\{U \times(X-\bar{U}): U \in \mathscr{W}\} \cup\{(X-\bar{U}) \times U: U \in \mathscr{W}\}
$$

covers $A \times B$. Since $A \times B$ is compact, there is a finite $\mathscr{E} \subset \mathscr{U}$ such that

$$
\{U \times(X-\bar{U}): U \in \mathscr{E}\} \cup\{X-\bar{U}) \times U: U \in \mathscr{E}\}
$$

covers $A \times B$. This $\mathscr{E}$ has the required property. ${ }^{3)}$

Henceforth, whenever $\mathscr{U}$ is a collection of subsets of $X$ and $x \in X$, we will write $\mathscr{U}(x)$ to denote $\{U \in \mathscr{C}: x \in U\}$.

LeMMA 3.4. Let $A, B$ be disjoint subsets of a set $X$, and $\mathscr{E}$ a finite collection of subsets of $X$ separating any $x \in A$ from any $x^{\prime} \in B$. Then some subcollection $\mathscr{F}$ of $\mathscr{E}$ covers $A$ but not $B$, or vice versa.

Proof. Suppose not. Then for each $x \in A$ there is an $x^{\prime} \in B$

${ }^{3}$ We are grateful to Roy Olson for pointing out an error in an earlier version of this proof. 
such that $\mathscr{E}\left(x^{\prime}\right) \subset \mathscr{E}(x)$, and vice versa. Hence there is a sequence $x_{n} \in X$, with $x_{n}$ alternately in $A$ and in $B$, such that $\mathscr{E}\left(x_{n+1}\right) \subset \mathscr{E}\left(x_{n}\right)$ for all $n$. Since $\mathscr{E}$ is finite, $\mathscr{E}\left(x_{n+1}\right)=\mathscr{E}\left(x_{n}\right)$ for some $n$, and that contradicts the separation property of $\mathscr{E}$.

4. Proof of Theorem 1.1. Let $f: X \rightarrow Y$ be continuous and onto, with $X$ an $E C$, and let us show that $Y$ must be an $E C$. By Theorem 1.4 (all of which except $(\mathrm{e}) \rightarrow(\mathrm{d})$ has been proved in $\S 2$ ), we may assume that $X$ satisfies 1.4(e), and we shall conclude that $Y$ satisfies 1.4(d). That will also establish the implication $1.4(\mathrm{e}) \rightarrow$ $1.4(\mathrm{~d})$.

Let $\mathscr{U}=\bigcup_{n=1}^{\infty} \mathscr{L}_{n}$ be an $F$-separating collection of open subsets of $X$, with each $\mathscr{U}_{n}$ point-finite; we may suppose that $\mathscr{U}_{n} \subset \mathscr{U}_{n+1}$ and that $\mathscr{U}_{n}$ covers $X$ for all $n$. Let $\Phi=\{\mathscr{F} \subset \mathscr{U}: \mathscr{F}$ finite $\}$. For each $\mathscr{F} \in \Phi$, let

$$
\begin{aligned}
& V(\mathscr{F})=\left\{y \in Y: \mathscr{F} \text { covers } f^{-1}(y)\right\} \\
& M(\mathscr{F})=\left\{y \in Y: \mathscr{F} \text { covers } f^{-1}(y) \text { minimally }\right\}
\end{aligned}
$$

Observe that $V(\mathscr{F})=Y-f(X-\cup \mathscr{F})$, so $V(\mathscr{F})$ is open in $Y$, and $M(\mathscr{F})=V(\mathscr{F})-\mathrm{U}\left\{V\left(\mathscr{F}^{\prime}\right): \mathscr{F}^{\prime} \subsetneq \mathscr{F}\right\}$, so $M(\mathscr{F})$ is relatively closed in $V(\mathscr{F})$.

For each $\mathscr{F} \in \Phi$, choose open $V_{\alpha}(\mathscr{F}) \subset V(\mathscr{F})$ and closed $S_{\alpha, m}(\mathscr{F}) \subset V_{\alpha}(\mathscr{F})(\alpha<\lambda=|Y|, m \in N)$ as in Lemma 3.2. Let

$$
M_{\alpha, m}(\mathscr{F})=S_{\alpha, m}(\mathscr{F}) \cap M(\mathscr{F}) \text {. }
$$

Then

$$
M(\mathscr{F})=\bigcup\left\{M_{\alpha, m}(\mathscr{F}): \alpha<\lambda, m \in N\right\},
$$

and each $M_{\alpha, m}(\mathscr{F})$ is closed in $Y$.

For $\mathscr{F} \in \Phi, \alpha<\lambda, m \in N$, we shall define a positive integer $k=k_{\alpha, m}(\mathscr{F})$ with the following property:

$$
\begin{aligned}
& \text { If } x, x^{\prime} \in X \text {, if } \mathscr{U}_{k}(x)=\mathscr{U}_{k}\left(x^{\prime}\right) \text {, and if } f(x) \in M_{\alpha, m}(\mathscr{F}) \text {, } \\
& \text { then } f\left(x^{\prime}\right) \in V_{\alpha}(\mathscr{F}) \text {. }
\end{aligned}
$$

To define $k$, let $A=f^{-1}\left(M_{\alpha, m}(\mathscr{F})\right), B=f^{-1}\left(Y-V_{\alpha}(\mathscr{F})\right)$. By Lemma 3.3, there is a finite $\mathscr{E} \subset \mathscr{U}$ which separates any $x \in A$ from any $x^{\prime} \in B$. Pick $k$ so that $\mathscr{E} \subset \mathscr{U}_{k}$; this $k$ satisfies (4.2).

Let $\mathscr{F} \in \Phi, \alpha<\lambda, m \in N$, and let $k$ be as above. Define

$$
\begin{aligned}
& H_{\alpha, m}(\mathscr{F})=\bigcup\left\{\cap\left(\mathscr{C}_{k}(x)\right): x \in f^{-1}\left(M_{\alpha, m}(\mathscr{F})\right)\right\}, \\
& K_{\alpha, m}(\mathscr{F})=Y-f\left(X-H_{\alpha, m}(\mathscr{F})\right) .
\end{aligned}
$$

Now $H_{\alpha, m}(\mathscr{F})$ is an open set in $X$ containing $f^{-1}\left(M_{\alpha, m}(\mathscr{F})\right)$, so 
$K_{\alpha, m}(\mathscr{F})$ is an open set in $Y$ containing $M_{\alpha, m}(\mathscr{F})$. Choose an open

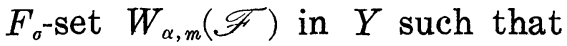

$$
M_{\alpha, m}(\mathscr{F}) \subset W_{\alpha, m}(\mathscr{F}) \subset\left(K_{\alpha, m}(\mathscr{F}) \cap V_{\alpha}(\mathscr{F})\right) .
$$

Finally, define

$$
\mathscr{W}^{n}=\left\{W_{\alpha, m}(\mathscr{F}): \mathscr{F} \in \Phi, \quad \alpha<\lambda, \quad m \in N\right\} .
$$

We will show that $\mathscr{W}$ is a $\sigma$-point-finite, separating collection of subsets of $Y$, thereby completing our proof.

(A) $\mathscr{W}$ is separating on $Y$ : Let $y \neq y^{\prime}$ be in $Y$. By Lemmas 3.3 and 3.4, there is a finite $\mathscr{F} \subset \mathscr{Q}$ which covers $f^{-1}(y)$ but not $f^{-1}\left(y^{\prime}\right)$, or vice versa (we won't repeat this phrase). We may assume that $\mathscr{F}$ is a minimal cover of $f^{-1}(y)$. Hence $y \in M(\mathscr{F})$ and $y^{\prime} \notin V(\mathscr{F})$. By (4.1), there are $\alpha<\lambda$ and $m \in N$ so that $y \in$ $M_{\alpha, m}(\mathscr{F})$. Hence $y \in W_{\alpha, m}(\mathscr{F})$ and $y^{\prime} \notin W_{\alpha, m}(\mathscr{F})$.

(B) $\mathscr{V}$ is $\sigma$-point-finite: For $m, n, k \in N$, let

$$
\begin{aligned}
& \mathscr{W}_{m, n, k}=\left\{W_{\alpha, m}(\mathscr{F}): \mathscr{F} \in \Phi, \mathscr{F} \subset \mathscr{U}_{n},|\mathscr{F}| \leqq n,\right. \\
&\left.k_{\alpha, m}(\mathscr{F})=k, \alpha<\lambda\right\} .
\end{aligned}
$$

Then $\mathscr{W}^{-}=\bigcup_{m, n, k=1}^{\infty} \mathscr{W}_{m, n, k}$, so we need only show that each $\mathscr{W}_{m, n, k}$ is point-finite.

Suppose some $\mathscr{W}_{m, n, k}$ were not point-finite. Then there are distinct $W_{\alpha_{j}, m}\left(\mathscr{F}_{j}\right)(j \in N)$ in $\mathscr{W}_{m, n, k}$, all containing the same $y^{*} \in Y$. Fix some $x^{*} \in f^{-1}\left(y^{*}\right)$. Then $x^{*} \in H_{\alpha_{j}, m}\left(\mathscr{F}_{j}\right)$, so there are $x_{j} \in$ $f^{-1}\left(M_{\alpha_{j}, m}\left(\mathscr{F}_{j}\right)\right)$ such that $x^{*} \in \bigcap \mathscr{U}_{k}\left(x_{j}\right)$ for all $j$. Hence $\mathscr{U}_{k}\left(x_{j}\right) \subset$ $\mathscr{U}_{k}\left(x^{*}\right)$ for all $j$; since $\mathscr{U}_{k}\left(x^{*}\right)$ is finite, we may assume, by passing to a subsequence, that $\mathscr{U}_{k}\left(x_{i}\right)=\mathscr{Q}_{k}\left(x_{j}\right)$ for all $i, j$. By (4.2), it follows that $f\left(x_{i}\right) \in V_{\alpha_{j}}\left(\mathscr{F}_{j}\right)$ for all $i, j$. Let $y_{i}=f\left(x_{i}\right)$. Then

$$
y_{i} \in M_{\alpha_{i}, m}\left(\mathscr{F}_{i}\right) \cap V_{\alpha_{j}}\left(\mathscr{F}_{j}\right) \quad(i, j \in N) .
$$

We now distinguish two cases to obtain our contradiction.

Case 1. There are $i \neq j$ with $\mathscr{F}_{i}=\mathscr{F}_{j}$ : Then $\alpha_{i} \neq \alpha_{j}$; suppose $\alpha_{i}>\alpha_{j}$ : Hence (4.3) implies that (writing $\mathscr{F}$ for $\mathscr{F}_{i}=\mathscr{F}_{j}$ )

$$
y_{i} \in S_{\alpha_{i}, m}(\mathscr{F}) \cap V_{\alpha_{j}}(\mathscr{F}) \text {, }
$$

contradicting condition (b) of Lemma 3.2.

Case 2. The $\mathscr{F}_{j}$ are all distinct: Let

$$
E=\bigcup_{j=1}^{\infty} f^{-1}\left(y_{j}\right) \text {. }
$$


Since $y_{i} \in V_{\alpha_{j}}\left(\mathscr{F}_{j}\right) \subset V\left(\mathscr{F}_{j}\right)$ for all $i$, $j$, each $\mathscr{F}_{j}$ convers $E$. Since $y_{j} \in M_{\alpha_{j}}\left(\mathscr{F}_{j}\right) \subset M\left(\mathscr{F}_{j}\right)$ for all $j$, each $\mathscr{F}_{j}$ if a minimal cover of $f^{-1}\left(y_{j}\right)$ and thus also of $E$. Hence $E$ has infinitely many minimal covers $\mathscr{F}_{j}$ with $\mathscr{F}_{j} \subset \mathscr{U}_{n}$ and $\left|\mathscr{F}_{j}\right| \leqq n$, contradicting Lemma 3.1.

That completes the proof.

5. Consequences of the proof of Theorem 1.1. The first consequence, which has already been observed in $\S 4$, is that 1.4 $(\mathrm{e}) \rightarrow 1.4(\mathrm{~d})$. This implication follows from the proof of Theorem 1.1 in the special case where $X=Y$ and $f=\mathrm{id}_{X}$, and, not surprisingly, the proof becomes somewhat simpler in this case: The only $\mathscr{F} \subset \mathscr{U}$ which now concern us are singletons, and if $\mathscr{F}=\{U\}$ then $V(\mathscr{F})=M(\mathscr{F})=U$. That $\mathscr{W}$ is separating is now trivial, as is Case 2 of the proof that $\mathscr{W}$ is $\sigma$-point-finite; hence Lemmas 3.4 and 3.1 are not needed here. The general structure of the proof is retained, however, and Lemmas 3.2 and 3.3 are apparently still required.

Our proof that $1.4(\mathrm{e}) \rightarrow 1.4(\mathrm{~d})$ reveals that this implication can be split into two stages, thereby obtaining yet another necessary and sufficient condition for a compact space $X$ to be an $E C$, as follows:

(5.1) X has a $\sigma$-point-finite collection $\mathscr{C}$ of open subsets such that, if $A, B$ are disjoint, closed subsets of $X$, then some finite $\mathscr{E} \subset \mathscr{Q}$ separates any $x \in A$ from any $x^{\prime} \in B$.

In fact, $1.4(\mathrm{e}) \rightarrow(5.1)$ by Lemma 3.3, and our proof of Theorem 1.1 shows that $(5.1) \rightarrow 1.4(\mathrm{~d})$.

We conclude this section by recording one more consequence of our proof of Theorem 1.1: Every open subset of an $E C$ is the union of a $\sigma$-point-finite collection of open $F_{\sigma}$ 's.

6. A modification of Eberlein compacts. Let $\Sigma(\Gamma)$ denote the space of real-valued functions on the set $\Gamma$ which vanish at all but countably many $\gamma \in \Gamma$, and let $\mathscr{T}_{p}$ be the topology of pointwise convergence on this space. Observe that $\Sigma(\Gamma) \supset c_{0}(\Gamma)$, and that $\left(\Sigma(\Gamma), \mathscr{T}_{p}\right)$ is just the $\Sigma$-product (in the sense of H.H. Corson [4]) of $|\Gamma|$-many real lines.

The proof of the following result is essentially the same as the proof of the equivalence of (c), (d) and (e) in Theorem 1.4, with the reference to Theorem 1.1 replaced by an analogous reference to Theorem 6.2 below.

THEOREM 6.1. The following properties of a compact space $X$ are equivalent. 
(a) $X$ is homeomorphic to a subset of some $\left(\Sigma(\Gamma), \mathscr{T}_{p}\right)$.

(b) $X$ has a point-countable, separating collection of open $F_{\sigma^{-}}$ subsets.

(c) $X$ has a point-countable, F-separating collection of open subsets.

Let us call a compact space satisfying the equivalent conditions of Theorem 6.1 a Corson compact (a term suggested by their relation to Corson's $\Sigma$-products). Observe that every Eberlein compact is a Corson compact and that, by Remark 1.7, it is consistent with $Z F C$ that the two concepts are distinct.

Analogously to Theorem 1.1, we now have:

THEOREM 6.2. Every continuous image of a Corson compact is again a Corson compact.

The proof of Theorem 6.2 is virtually the same as that of Theorem 1.1, except that Lemma 3.1 must be replaced by the following result.

Lemma 6.3 (A. S. Miščenko [7]). Let $\mathscr{P}$ be a point-countable collection of subsets of $X$, and let $E \subset X$. Then there are at most countably many minimal finite covers $\mathscr{F}$ of $E$ with $\mathscr{F} \subset \mathscr{P}$.

Added in Proof. The second author has shown that the answer to Problem 1.8 is "yes" if $X$ is a closed subset of $\left(c_{0}(\Gamma), \mathscr{T}_{p}\right)$.

\section{REFERENCES}

1. D. Amir and J. Lindenstrauss, The structure of weakly compact sets in Banach spaces, Ann. of Math., 88 (1968), 34-46.

2. Y. Benyamini, M. E. Rudin and M. Wage, Continuous images of weakly compact subsets of Banach spaces, to appear in Pacific J. Math.

3. D. Burke, Preservation of certain base axioms under a perfect mapping, Proceedings of the Auburn Conference in General Topology, 1976.

4. H. H. Corson, Normality in subsets of product spaces, Amer. J. Math., 81 (1959), 785-796.

5. A. Grothendieck, Critères de compacité dans les espaces fonctionels generaux, Amer.

J. Math., 74 (1952), 168-186.

6. J. Lindenstrauss, Weakly compact sets-their topological properties and the Banach spaces they generate, Annals of Mathematics Studies 69, Princeton Univ. Press (1972), 235-273.

7. A. S. Miščenko, Spaces with a pointwise denumerable basis, Dokl. Akad Nauk SSSR, 145 (1962), 985-988. (Soviet Math. Dokl. 3 (1962), 855-858).

8. J. Nagata, A note on Filipov's theorem, Proc. Japan Acad., 45 (1969), 30-33.

9. H. P. Rosenthal, The heredity problem for weakly compactly generated Banach spaces, Compositio Math., 28 (1974), 83-111. 
10. P. Simon, On continuous images of Eberlein compacts, Comm. Math. Carolinae, 17 (1976), 179-194.

11. M. Wage, Applications of set theory to analysis and topology, Thesis, University of Wisconsin, 1976.

Received February 24, 1977.

UNIVERSITY OF WASHINGTON

Seattle, WA 98195

AND

UNIVERSITY OF WISCONSIN

MADISON, WI 53706 



\title{
PACIFIC JOURNAL OF MATHEMATICS
}

\section{EDITORS}

\author{
RICHARD ARENS (Managing Editor) \\ University of California \\ Los Angeles, CA 90024 \\ Charles W. Curtis \\ University of Oregon \\ Eugene, OR 97403 \\ C. C. MOORE \\ University of California \\ Berkeley, CA 94720
}

\section{J. DUGUNDJI}

Department of Mathematics

University of Southern California

Los Angeles, CA 90007

R. FINN and J. MILGRAM

Stanford University

Stanford, CA 94305

\section{ASSOCIATE EDITORS}
E. F. BECKENBACH
B. H. NeumanN
F. WOLF
K. YoSHIDA

\section{SUPPORTING INSTITUTIONS}

UNIVERSITY OF BRITISH COLUMBIA

CALIFORNIA INSTITUTE OF TECHNOLOGY

UNIVERSITY OF CALIFORNIA

MONTANA STATE UNIVERSITY

UNIVERSITY OF NEVADA, RENO

NEW MEXICO STATE UNIVERSITY

OREGON STATE UNIVERSITY

UNIVERSITY OF OREGON

OSAKA UNIVERSITY

\author{
UNIVERSITY OF SOUTHERN CALIFORNIA \\ STANFORD UNIVERSITY \\ UNIVERSITY OF HAWAII \\ UNIVERSITY OF TOKYO \\ UNIVERSITY OF UTAH \\ WASHINGTON STATE UNIVERSITY \\ UNIVERSITY OF WASHINGTON \\ $\stackrel{*}{*} \stackrel{*}{*} \stackrel{*}{*}$ AMERICAN MATHEMATICAL SOCIETY
}

The Supporting Institutions listed above contribute to the cost of publication of this Journal, but they are not owners or publishers and have no responsibility for its content or policies.

Mathematical papers intended for publication in the Pacific Jaurnal of Mathematics should be in typed form or offset-reproduced, (not dittoed), double spaced with large margins. Please do not use built up fractions in the text of your manuscript. You may however, use them in the displayed equations. Underline Greek letters in red, German in green, and script in blue. The first paragraph or two must be capable of being used separately as a synopsis of the entire paper. Items of the bibliography should not be cited there unless absolutely necessary, in which case they must be identified by author and Journal, rather than by item number. Manuscripts, in triplicate, may be sent to any one of the editors. Please classify according to the scheme of Math. Reviews, Index to Vol. 39. All other communications should be addressed to the managing editor, or Elaine Barth, University of California, Los Angeles, California, 90024.

The Pacific Journal of Mathematics expects the author's institution to pay page charges, and reserves the right to delay publication for nonpayment of charges in case of financial emergency.

100 reprints are provided free for each article, only if page charges have been substantially paid. Additional copies may be obtained at cost in multiples of 50 .

The Pacific Journal of Mathematics is issued monthly as of January 1966. Regular subscription rate: $\$ 7200$ a year (6 Vols., 12 issues). Special rate: $\$ 36.00$ a year to individual members of supporting institutions.

Subscriptions, orders for back numbers, and changes of address should be sent to Pacific Journal of Mathematics, 103 Highland Boulevard, Berkeley, California, 94708.

PUBLISHED BY PACIFIC JOURNAL OF MATHEMATICS, A NON-PROFIT CORPORATION

Printed at Kokusai Bunken Insatsusha (International Academic Printing Co., Ltd.). 8-8, 3-chome, Takadanobaba, Shinjuku-ku, Tokyo 160, Japan.

Copyright (C) 1975 by Pacific Journal of Mathematics Manufactured and first issued in Japan 


\section{Pacific Journal of Mathematics}

Vol. 72, No. 2

February, 1977

George E. Andrews, Plane partitions. II. The equivalence of the

Bender-Knuth and MacMahon conjectures ................. 283

Lee Wilson Badger, An Ehrenfeucht game for the multivariable quantifiers

of Malitz and some applications ......................... 293

Wayne C. Bell, A decomposition of additive set functions ............ 305

Bruce Blackadar, Infinite tensor products of $C^{*}$-algebras ............. 313

Arne Brøndsted, The inner aperture of a convex set .............. 335

N. Burgoyne, Finite groups with Chevalley-type components........... 341

Richard Dowell Byrd, Justin Thomas Lloyd and Roberto A. Mena, On the retractability of some one-relator groups .....................

Paul Robert Chernoff, Schrödinger and Dirac operators with singular potentials and hyperbolic equations .................... 361

John J. F. Fournier, Sharpness in Young's inequality for convolution ....... 383

Stanley Phillip Franklin and Barbara V. Smith Thomas, On the metrizability

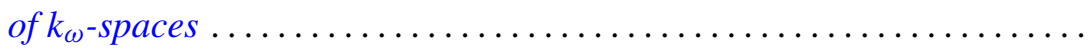

David Andrew Gay, Andrew McDaniel and William Yslas Vélez, Partially normal radical extensions of the rationals .................... 403

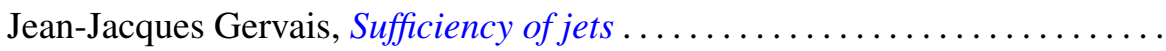

Kenneth R. Goodearl, Completions of regular rings. II . .

Sarah J. Gottlieb, Algebraic automorphisms of algebraic groups with stable maximal tori

Donald Gordon James, Invariant submodules of unimodular Hermitian forms.....

J. Kyle, $W_{\delta}(T)$ is convex.

Ernest A. Michael and Mary Ellen Rudin, A note on Eberlein compacts ...

Ernest A. Michael and Mary Ellen Rudin, Another note on Eberlein compacts ....

Thomas Bourque Muenzenberger and Raymond Earl Smithson, Fixed point theorems for acyclic and dendritic spaces.

Budh Singh Nashier and A. R. Rajwade, Determination of a unique solution of the quadratic partition for primes $p \equiv 1(\bmod 7)$.

Frederick J. Scott, New partial asymptotic stability results for nonlinear ordinary differential equations ....................

Frank Servedio, Affine open orbits, reductive isotropy groups, and dominant gradient morphisms; a theorem of Mikio Sato..........

D. Suryanarayana, On the distribution of some generalized square-full integers.................................. 